\title{
Study of Second Grade Fluid over a Rotating Disk with Coriolis and Centrifugal Forces
}

\section{Shuaiba $M^{*}$, Shaha RA and Khana A}

Department of Basic Sciences and Islamiat, University of Engineering and Technology, Pakistan

\begin{abstract}
The steady flow of an incompressible second grade viscoelastic fluid above a rotating disk that is stretching in radial direction is studied. The Coriolis and centrifugal forces are taking into account. The constitutive non-linear partial differential equations are transformed to system of ordinary differential equations. For analysis Homotopy Analysis Metod (HAM) BVPh2.0 package is used. The effects of viscoelastic parameter $\alpha$, rotating parameter $\beta$, stretching parameter $\mathrm{\gamma}$, Coriolis and centrifugal forces on the velocity component are discussed.
\end{abstract}

Keywords: Rotating disk; Free surface flow; Rotating starching disk; HAM mathematica package

\section{Introduction}

Rotating disk flow plays an important role in the field of engineering and industry. Centrifugal pumps are extensively used in petroleum industry to transport high viscosity fluids such as waxy crude oils. The first study of rotating disk was introduced by Von Karman in 1921 [1]. He was able to found that the rotating disk flow is a type of boundary layer flow and there is no depend of radial distance on the boundary thickness. In 1934, cochran [2], provided asymptotic solution to the ordinary differential equations derived by Von Karman. Although the analysis was simple but valuable in the field of rotating field. The work of cochran was extended by Benton [3], in 1966. He provided better solutions and solved the unsteady problem.

In recent years, much attention has been given to the rotating flow of non-Newtonian fluids concerning to its applications in industries. The steady flow of non-Newtonian fluid over rotating disk with uniform suction was considered by Mithal [4], in 1961. His solutions were valid for small values of non-Newtonian problems.

Later, Attia [5] in 2003 extended the idea of Mithal and studied the same problem to the transient state with heat transfer. Their solutions were valid for the whole range of the parameters. In addition the reader may consult $[6,7]$ for the studies of non-Newtonian fluids.

Boundary layer flow equations are developed by Reynolds number $\mathrm{Re} \rightarrow \infty$ in the boundary layer region combine with the use of the order of $\varepsilon^{2} \operatorname{Re} \approx 1$, where $\varepsilon^{2} \rightarrow 0$.

A challenging mathematical model is developed with the nonlinearity in the term involving maximum order derivation. Most of analytic methods such as Adomian Decomposition Method, Differential Transform Method, Variation Iterative Method and Optimal Homotapy Asymptotic Methods fails to solve this problem. We handle this problem by HAM BVPh2.0 package [8] using 20thorder of approximations.

The following strategy is applied to the rest of the paper. In section 3 the basic governing equations for the motion are formulated in cylindrical coordinates. Section 4 is the solution by homotopy analysis method. Section 5 is the error analysis. Section 6 contains the numerical results and their discussion for different values of physical parameters. Finally, our conclusion follows in section 7.

\section{Formulation of the Problem}

Let us consider the steady incompressible flow of a Rivlin-Erickson type fluid produced by the rotation of an insulated disk of radius $R$ with angular speed $\Omega$ and radial stretching. The disk is stretching in radial direction which has a velocity $u_{\omega}(r)$. The co-ordinate system $(r, \theta, z)$ is adopted whose origin is taken at the center of the disk. In which $r$-axis is along the radius of the disk, $\mathrm{z}$-axis is perpendicular to the disk and $\theta$ is oriented in the direction of rotation. Assuming flow is laminar, axisymetric and its density $\rho$, is constant.

\section{Boundary conditions}

$$
\begin{aligned}
& \tilde{u}=A r ; \quad \tilde{v}=\Omega r ; \quad \tilde{w}=0 \quad \text { atz }=0 \\
& \tilde{u} \rightarrow 0 ; \quad \tilde{v} \rightarrow 0 \quad \text { asz } \rightarrow \infty
\end{aligned}
$$

Due to no penetration the value of $\tilde{w}$ vanishes near the surface of the insulated disk. The tangential velocity $\tilde{v}$ have a value $\Omega \mathrm{r}$ at the disk surface. The position vector is given by

$$
\bar{r}=(r \cos \theta, r \sin \theta, z)
$$

The velocity vector is $\bar{\omega}=(0,0, \Omega)$.

The basic equations governing the flow of second grade fluid is the continuity equation

$$
\overleftarrow{\nabla} \cdot \bar{u}=0
$$

and Navier-Stokes equations (NSE)

$$
\rho\left[\frac{D \bar{V}}{D t}+2 \bar{w} \times \bar{V}+\bar{w} \times(\bar{w} \times \bar{r})\right]=-\bar{\nabla} \bar{p}+\bar{\nabla} \cdot \bar{\tau}
$$

where $\frac{D}{D t}$ is material derivative $\rho$ is constant density, $\bar{p}$ is dynamic pressure, $\bar{\tau}$ is shear stress, $\bar{r}$ is the position vector $\bar{V}$ is velocity vector and $\tilde{w}$ is the rotational vector given as

${ }^{*}$ Corresponding author: Shuaiba M, Department of Basic Sciences and Islamiat University of Engineering and Technology, Pakistan, Tel: 444454548; E-mail: mshoaib01@yahoo.com

Received July 26, 2017; Accepted August 28, 2017; Published September 03 2017

Citation: Shuaiba M, Shaha RA, Khana A (2017) Study of Second Grade Fluid over a Rotating Disk with Coriolis and Centrifugal Forces. J Phys Math 8: 242. doi: 10.4172/2090-0902.1000242

Copyright: @ 2017 Shuaiba M, et al. This is an open-access article distributed under the terms of the Creative Commons Attribution License, which permits unrestricted use, distribution, and reproduction in any medium, provided the original author and source are credited. 


$$
\bar{r}=\left(\begin{array}{l}
r \cos \theta \\
r \sin \theta \\
z
\end{array}\right), \bar{V}=\left(\begin{array}{l}
\tilde{u} \\
\tilde{v} \\
\tilde{w}
\end{array}\right), \bar{w}=\left(\begin{array}{l}
0 \\
0 \\
\Omega
\end{array}\right)
$$

where $r$ is the radius, $\theta$ is the angle and $\mathrm{z}$ is a wall normal co-ordinate, $\tilde{u}, \tilde{v}, \tilde{w}$ are radial, azimuthal and axial velocity components respectively. The continuity equation for incompressible fluid is

$$
\frac{\partial \tilde{u}}{\partial r}+\frac{\partial \tilde{v}}{r}+\frac{\partial \tilde{w}}{\partial z}+\frac{\tilde{u}}{r}=
$$

Equation (3) becomes

$$
\underbrace{\rho\left[\frac{\partial v}{\partial t}+(v \cdot \nabla) v\right.}_{1}+\underbrace{2 \bar{w} \times \bar{V}}_{2}+\underbrace{\bar{w} \times(\bar{w} \times \bar{r})]}_{3}=\underbrace{-\nabla p}_{4}+\underbrace{\bar{\nabla} \cdot \bar{\tau}}_{5}
$$

Where 1 is the material differential term. The Coriolis force terms 2 given as

$$
2 \bar{w} \times \bar{v}=\left(\begin{array}{l}
-2 \tilde{v} \Omega \\
2 \tilde{u} \Omega \\
0
\end{array}\right)
$$

the centrifugal force term 3 is

$$
\bar{w} \times(\bar{w} \times \bar{r})=\grave{U}^{2}\left(\begin{array}{l}
-r \cos \theta \\
-r \sin \theta \\
0
\end{array}\right)
$$

The pressure term 4 in component form is

$$
\left(\begin{array}{l}
0.2 \mathrm{~cm} \frac{\partial p}{\partial r} \\
0.2 \mathrm{~cm} \frac{1}{r} \frac{\partial p}{\partial \theta} \\
\frac{\partial p}{\partial z}
\end{array}\right)
$$

and 5 is stress tensor in a second grade fluid given as

$$
\bar{\tau}=\bar{A}_{1}+\alpha_{1} \bar{A}_{2}+\alpha_{2} \bar{A}_{1}^{2}
$$

Where $\alpha_{1} \alpha_{1}$ are material constants and $\overline{A_{1}}$ and $\overline{A_{2}}$ are RivlinEricksen tensors, given as

$$
\begin{aligned}
& \bar{A}_{1}=\bar{L}+\bar{L}^{T} ; \bar{L}=\bar{\nabla} \cdot \bar{u} \\
& \overleftarrow{A_{2}}=\frac{d \bar{A}_{1}}{d t}+\overline{A_{1}}+\overline{A_{1}} \bar{L}+\bar{L}^{T} \bar{A}_{1}
\end{aligned}
$$

The continuity equation in cylindrical coordinates has the form

$$
\frac{1}{r} \frac{\partial(r \tilde{u})}{\partial r}+\frac{\partial \tilde{w}}{\partial z}=0
$$

Momentum equations in cylindrical co-ordinates are r-component of momentum equation:

$$
\rho\left[\frac{\partial \tilde{\partial} u}{\partial t}+\tilde{u} \frac{\partial \tilde{u}}{\partial r}+\frac{\tilde{v}}{r} \frac{\tilde{u}}{\partial \theta}-\frac{\tilde{w} \tilde{v}}{r}+\tilde{w} \frac{\partial \tilde{u}}{\partial z}-2 \tilde{\tilde{v}} \Omega-r \Omega^{2}\right]=-\frac{\partial \tilde{p}}{\partial r}+\left[\frac{1}{r} \frac{\partial\left(r \tau_{r r}\right)}{\partial r}+\frac{1}{r} \frac{\partial \tau_{r \theta}}{\partial \theta}+\frac{\partial \tau_{r z}}{\partial z}-\frac{1}{r} \tau_{\theta \theta}\right]
$$

$\theta$-component of momentum equation:

$$
\rho\left[\frac{\partial \tilde{v}}{\partial t}+\tilde{u} \frac{\partial \tilde{v}}{\partial r}+\frac{\tilde{v}}{r} \frac{\tilde{v}}{\partial \theta}-\frac{\tilde{u} \tilde{v}}{r}+\tilde{w} \frac{\partial \tilde{v}}{\partial z}+2 \tilde{u} \Omega\right]=-\frac{1}{r} \frac{\partial \tilde{p}}{\partial \theta}+\left[\frac{1}{r^{2}} \frac{\partial\left(r^{2} \tau_{\theta r}\right)}{\partial r}+\frac{1}{r} \frac{\partial \tau_{\theta \theta}}{\partial \theta}+\frac{\partial \tau_{\theta z}}{\partial z}-\frac{\tau_{\theta r}-\tau_{r \theta}}{r}\right]
$$

z-component of momentum equation:

$$
\rho\left[\frac{\partial \tilde{w}}{\partial t}+\tilde{u} \frac{\partial \tilde{w}}{\partial r}+\frac{\tilde{v}}{r} \frac{\tilde{w}}{\partial \theta}+\tilde{w} \frac{\partial \tilde{w}}{\partial z}\right]=-\frac{\partial \tilde{p}}{\partial z}+\left[\frac{1}{r} \frac{\partial\left(r \tau_{z r}\right)}{\partial r}+\frac{1}{r} \frac{\partial \tau_{z \theta}}{\partial \theta}+\frac{\partial \tau_{z z}}{\partial z}\right]
$$

After making use of assumptions and shear stress eqns. (10-12) takes the form

$$
\begin{aligned}
& \rho\left[\tilde{u} \frac{\partial \tilde{u}}{\partial r}-\frac{\tilde{v}^{2}}{r}+\tilde{w} \frac{\partial \tilde{u}}{\partial z}-2 \tilde{v} \Omega-r \Omega^{2}\right]=-\frac{\partial \tilde{p}}{\partial r}+\mu\left[\frac{2}{r} \frac{\partial \tilde{u}}{\partial r}+2 \frac{\partial^{2} \tilde{u}}{\partial r^{2}}+\frac{\partial^{2} \tilde{w}}{\partial z \partial \partial}+\frac{\partial^{2} \tilde{u}}{\partial z^{2}}-\frac{2 \tilde{u}}{r^{2}}\right] \\
& +\alpha_{1}\left[\frac{2 \tilde{v}}{r^{2}} \frac{\partial \tilde{v}}{\partial r}+\frac{1}{r}\left(\frac{\partial \tilde{u}}{\partial z}\right)^{2}+2 \frac{\partial \tilde{u}}{\partial z} \frac{\partial^{2} \tilde{u}}{\partial z \partial r}-\frac{1}{r}\left(\frac{\partial \tilde{v}}{\partial r}\right)^{2}\right. \\
& -2 \frac{\partial \tilde{v}}{\partial r} \frac{\partial^{2} \tilde{v}}{\partial r^{2}}-\frac{1}{r}\left(\frac{\partial \tilde{w}}{\partial r}\right)^{2}-2 \frac{\partial \tilde{w}}{\partial r} \frac{\partial^{2} \tilde{w}}{\partial r^{2}}+2 \tilde{u} \frac{\partial^{3} \tilde{u}}{\partial r^{3}}+2 \frac{\tilde{u}}{r} \frac{\partial^{2} \tilde{u}}{\partial r^{2}} \\
& +2 \frac{\partial \tilde{u}}{\partial r} \frac{\partial^{2} \tilde{u}}{\partial r^{2}}+2 \tilde{w} \frac{\partial^{3} \tilde{u}}{\partial z \partial r^{2}}+2 \frac{\tilde{w}}{r} \frac{\partial^{2} \tilde{u}}{\partial z \partial r}+2 \frac{\partial \tilde{w}}{\partial r} \frac{\partial^{2} \tilde{u}}{\partial r \partial z} \\
& -\frac{\partial \tilde{w}}{\partial r} \frac{\partial^{2} \tilde{w}}{\partial z^{2}}-\frac{\partial \tilde{w}}{\partial z} \frac{\partial^{2} \tilde{w}}{\partial r \partial z}+\frac{\partial^{2} \tilde{w}}{\partial z^{2}} \frac{\partial \tilde{u}}{\partial r}+\frac{\partial^{2} \tilde{u}}{\partial z^{2}} \frac{\partial \tilde{w}}{\partial z}-\frac{\partial u}{\partial r} \frac{\partial^{2} \tilde{u}}{\partial z^{2}} \\
& -\frac{\partial \tilde{u}}{\partial} \frac{\partial^{2} \tilde{u}}{\partial t r}-\frac{\partial \tilde{v}}{\partial z} \frac{\partial^{2} \tilde{v}}{\partial t}-\frac{\partial \tilde{v}}{\partial} \frac{\partial^{2} \tilde{v}}{\partial z^{2}}+\frac{\partial \tilde{u}}{\partial} \frac{\partial^{2} \tilde{w}}{\partial z}+\frac{\partial \tilde{w}}{\partial} \frac{\partial^{2} \tilde{u}}{\partial t r}
\end{aligned}
$$

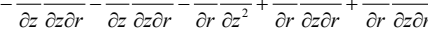

$$
\begin{aligned}
& +\tilde{u} \frac{\partial^{3} \tilde{w}}{\partial z \partial r^{3}}+\frac{\partial \tilde{u}}{\partial z} \frac{\partial^{2} \tilde{w}}{\partial r^{2}}+\tilde{u} \frac{\partial^{3} \tilde{u}}{\partial z^{2} \partial r}+\frac{\partial \tilde{u}}{\partial z} \frac{\partial^{2} \tilde{u}}{\partial z \partial r}+\frac{\tilde{v}}{r} \frac{\partial^{3} \tilde{u}}{\partial z^{3}} \\
& +\tilde{w} \frac{\partial^{3} \tilde{w}}{\partial z^{2} \partial r}+\frac{\partial \tilde{w}}{\partial z} \frac{\partial^{2} \tilde{w}}{\partial z \partial \partial}+\tilde{w} \frac{\partial^{3} \tilde{u}}{\partial z^{3}}+\frac{\partial \tilde{w}}{\partial z} \frac{\partial^{2} \tilde{u}}{\partial z^{2}}-\frac{1}{r}\left(\frac{\partial \tilde{u}}{\partial r}\right)^{2} \\
& \left.-\frac{1}{r}\left(\frac{\partial \tilde{v}}{\partial z}\right)^{2}-\frac{2 \tilde{u}}{r^{2}} \frac{\partial \tilde{u}}{\partial r}+2 \frac{\tilde{u}^{2}}{r^{3}}-2 \frac{\tilde{w}}{r^{2}} \frac{\partial \tilde{u}}{\partial z}\right] \\
& \rho\left[\tilde{u} \frac{\partial \tilde{v}}{\partial r}+\frac{\tilde{u} \tilde{v}}{r}+\tilde{w} \frac{\partial \tilde{v}}{\partial z}+2 \tilde{u} \Omega\right]=-\frac{1}{r} \frac{\partial \tilde{p}}{\partial \theta}+\mu\left[\frac{2}{r} \frac{\partial \tilde{v}}{\partial r}+\frac{\partial^{2} \tilde{v}}{\partial r^{2}}-\frac{1}{r} \frac{\partial \tilde{v}}{\partial r}-\frac{\tilde{v}}{r^{2}}+\frac{\partial^{2} \tilde{v}}{\partial z^{2}}\right] \\
& +\alpha_{1}\left[2 \frac{\partial \tilde{u}}{\partial r} \frac{\partial^{2} \tilde{v}}{\partial r^{2}}+\frac{\partial^{2} \tilde{u}}{\partial r^{2}} \frac{\partial \tilde{v}}{\partial r}+\frac{\tilde{v}}{r} \frac{\partial^{2} \tilde{u}}{\partial r^{2}}+\frac{\tilde{v}}{r^{2}} \frac{\partial \tilde{u}}{\partial r}+\frac{1}{r} \frac{\partial \tilde{u}}{\partial r} \frac{\partial \tilde{v}}{\partial r}\right. \\
& -\frac{2 \tilde{u}}{r} \frac{\partial^{2} \tilde{v}}{\partial r^{2}}-\frac{2 u}{r^{2}} \frac{\partial \tilde{v}}{\partial r}+\frac{2}{r} \frac{\partial \tilde{v}}{\partial z} \frac{\partial \tilde{u}}{\partial z}+\frac{\partial \tilde{v}}{\partial z} \frac{\partial^{2} \tilde{u}}{\partial r \partial z}+\frac{\partial^{2} \tilde{v}}{\partial r \partial z}+\tilde{u} \frac{\partial^{3} \tilde{v}}{\partial r^{3}} \\
& +\frac{2 \tilde{u}}{r} \frac{\partial^{2} \tilde{v}}{\partial r^{2}}+\tilde{w} \frac{\partial^{3} \tilde{v}}{\partial r^{2} \partial z}+\frac{2 \tilde{w}}{r} \frac{\partial^{2} \tilde{v}}{\partial r \partial z}+\frac{\partial \tilde{w}}{\partial r} \frac{\partial^{2} \tilde{v}}{\partial z \partial r}-\frac{\tilde{w}}{r} \frac{\partial^{2} \tilde{v}}{\partial z \partial r} \\
& -\frac{w}{r^{2}} \frac{\partial \tilde{v}}{\partial z}-\frac{1}{r} \frac{\partial \tilde{w}}{\partial r} \frac{\partial \tilde{v}}{\partial z}+\frac{\partial \tilde{v}}{\partial z} \frac{\partial^{2} \tilde{w}}{\partial z^{2}}+\frac{\partial \tilde{w}}{\partial z} \frac{\partial^{2} \tilde{v}}{\partial z^{2}}+\frac{\partial \tilde{v}}{\partial r} \frac{\partial^{2} \tilde{w}}{\partial z \partial r} \\
& +\frac{\partial \tilde{w}}{\partial r} \frac{\partial^{2} \tilde{v}}{\partial z \partial r}-\frac{\tilde{u}}{r} \frac{\partial^{2} \tilde{v}}{\partial z^{2}}-\frac{1}{r} \frac{\partial \tilde{u}}{\partial z} \frac{\partial \tilde{v}}{\partial z}+\frac{\tilde{v}}{r} \frac{\partial^{2} \tilde{u}}{\partial z^{2}}+\frac{1}{r} \frac{\partial \tilde{v}}{\partial z} \frac{\partial \tilde{u}}{\partial z} \\
& \left.+\tilde{u} \frac{\partial^{3} \tilde{v}}{\partial z^{2} \partial r}+\frac{\partial \tilde{u}}{\partial z} \frac{\partial^{2} \tilde{v}}{\partial r \partial z}+\tilde{w} \frac{\partial^{3} \tilde{v}}{\partial z^{3}}+\frac{\partial \tilde{w}}{\partial z} \frac{\partial^{2} \tilde{v}}{\partial z^{2}}\right] \\
& \rho\left[\tilde{u} \frac{\partial \tilde{w}}{\partial r}+\tilde{w} \frac{\partial \tilde{w}}{\partial z}\right]=-\frac{\partial \tilde{p}}{\partial z}+\mu\left[\frac{\partial^{2} \tilde{w}}{\partial r^{2}}+\frac{1}{r} \frac{\partial \tilde{w}}{\partial r}+\frac{\partial^{2} \tilde{u}}{\partial z \partial r}+\frac{1}{r} \frac{\partial \tilde{u}}{\partial z}+2 \frac{\partial^{2} \tilde{w}}{\partial z^{2}}\right] \\
& +\alpha_{1}\left[\frac{\partial \tilde{w}}{\partial r} \frac{\partial^{2} \tilde{u}}{\partial r^{2}}+\frac{\partial^{2} \tilde{w}}{\partial r^{2}} \frac{\partial \tilde{u}}{\partial r}+\frac{1}{r} \frac{\partial \tilde{w}}{\partial r} \frac{\partial \tilde{u}}{\partial r}-\frac{\partial \tilde{u}}{\partial r} \frac{\partial^{2} \tilde{u}}{\partial r \partial z}-\frac{\partial^{2} \tilde{u}}{\partial r^{2}} \frac{\partial \tilde{u}}{\partial z}-\frac{1}{r} \frac{\partial \tilde{u}}{\partial r} \frac{\partial \tilde{u}}{\partial z}\right. \\
& +\frac{\partial \tilde{w}}{\partial z} \frac{\partial^{2} \tilde{u}}{\partial r \partial z}+\frac{\partial \tilde{u}}{\partial z} \frac{\partial^{2} \tilde{w}}{\partial r \partial z}+\frac{1}{r} \frac{\partial \tilde{w}}{\partial z} \frac{\partial \tilde{u}}{\partial z}-\frac{\partial^{2} \tilde{w}}{\partial r \partial z} \frac{\partial \tilde{w}}{\partial r}-\frac{\partial^{2} \tilde{w}}{\partial r^{2}} \frac{\partial \tilde{w}}{\partial z}-\frac{1}{r} \frac{\partial \tilde{w}}{\partial r} \frac{\partial \tilde{w}}{\partial z} \\
& -\frac{\partial \tilde{v}}{\partial r} \frac{\partial^{2} \tilde{v}}{\partial r \partial z}-\frac{\partial^{2} \tilde{v}}{\partial r^{2}} \frac{\partial^{2} \tilde{v}}{\partial z^{2}}-\frac{1}{r} \frac{\partial \tilde{v}}{\partial r} \frac{\partial \tilde{v}}{\partial z}+\tilde{u} \frac{\partial^{3} \tilde{u}}{\partial r^{2} \partial z}+\frac{\tilde{u}}{r} \frac{\partial^{2} \tilde{u}}{\partial r \partial z}+\frac{\partial \tilde{u}}{\partial r} \frac{\partial^{2} \tilde{u}}{\partial r \partial z} \\
& +\tilde{u} \frac{\partial^{3} \tilde{w}}{\partial r^{3}}+\frac{\tilde{u}}{r} \frac{\partial^{2} \tilde{w}}{\partial r^{2}}+\frac{\partial^{2} \tilde{w}}{\partial r^{2}} \frac{\partial \tilde{u}}{\partial r}+\tilde{w} \frac{\partial^{3} \tilde{u}}{\partial r \partial z^{2}}+\frac{\tilde{w}}{r} \frac{\partial^{2} \tilde{u}}{\partial z^{2}}+\frac{\partial \tilde{w}}{\partial r} \frac{\partial^{2} \tilde{u}}{\partial z^{2}}+\tilde{w} \frac{\partial^{3} \tilde{w}}{\partial z \partial r^{2}} \\
& +\frac{\tilde{w}}{r} \frac{\partial^{2} \tilde{w}}{\partial r \partial z}+\frac{\partial \tilde{w}}{\partial r} \frac{\partial^{2} \tilde{w}}{\partial r \partial z}+2 \frac{\partial \tilde{w}}{\partial r} \frac{\partial^{2} \tilde{w}}{\partial z \partial r}-2 \frac{\partial \tilde{u}}{\partial z} \frac{\partial^{2} \tilde{u}}{\partial z^{2}}-2 \frac{\partial \tilde{v}}{\partial z} \frac{\partial^{2} \tilde{v}}{\partial z^{2}}+2 \tilde{u} \frac{\partial^{3} \tilde{w}}{\partial r \partial z^{2}} \\
& \left.+2 \frac{\partial \tilde{u}}{\partial z} \frac{\partial^{2} \tilde{w}}{\partial r \partial z}+2 \tilde{w} \frac{\partial^{3} \tilde{w}}{\partial z^{3}}+2 \frac{\partial \tilde{w}}{\partial z} \frac{\partial^{2} \tilde{w}}{\partial z^{2}}\right]
\end{aligned}
$$

Radial co-ordinate is scaled on a characteristic length along the radius of disk $\mathrm{R}$, and the surface normal co-ordinate is further scaled on boundary layer thickness $\delta=\left(\frac{v}{\Omega}\right)^{\frac{1}{2}}$.

$$
r^{*}=\frac{r}{R}, \quad z^{*}=\frac{z}{\left(\frac{v}{\Omega}\right)^{\frac{1}{2}}}
$$

The radial and azimuthal velocity components are nondimensionalized using the local surface velocity $\Omega R$, and the axial velocity component is non-dimensionalized with the local boundary layer angular velocity (Figure 1). 


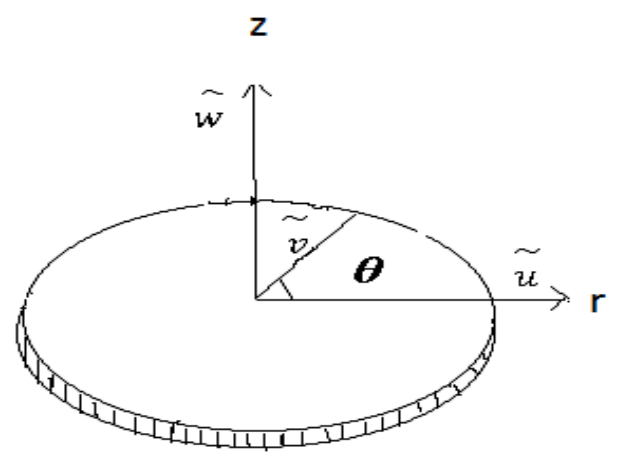

Figure 1: Geometrical representation of the ow over a radially stretching rotating disk

$$
u^{*}=\frac{\tilde{u}}{\Omega R}, \quad v^{*}=\frac{\tilde{v}}{\Omega R}, \quad w^{*}=\frac{\tilde{w}}{\delta \Omega}
$$

We scale the pressure as

$$
p^{*}=\frac{\tilde{p}}{\rho \Omega^{2} R^{2}}
$$

Particular choices of above dimensionless values leads to the Reynolds number

$$
R e=\frac{\rho \Omega \delta^{2}}{\mu}
$$

Using eqns. (16-19) and making use of $\varepsilon^{2} R e=O(1)$, the continuity equation (9) and momentum equations (13-15), after dropping asterisks, we obtain

$$
\begin{aligned}
& \frac{1}{r} \frac{\partial(r u)}{\partial r}+\frac{\partial w}{\partial z}=0 \\
& {\left[u \frac{\partial u}{\partial r}-\frac{v^{2}}{r}+w \frac{\partial u}{\partial z}\right]=\frac{\partial^{2} u}{\partial z^{2}}+\Omega \delta^{2} v(2 v+r)+\frac{\alpha_{1} \Omega}{\mu}\left[\frac{1}{r}\left(\frac{\partial u}{\partial z}\right)^{2}+\frac{\partial u}{\partial z} \frac{\partial^{2} u}{\partial z \partial r}\right.} \\
& +\frac{\partial u}{\partial z} \frac{\partial^{2} w}{\partial z^{2}}+\frac{\partial w}{\partial z} \frac{\partial^{2} u}{\partial z^{2}}-\frac{\partial u}{\partial r} \frac{\partial^{2} u}{\partial z^{2}}-\frac{\partial v}{\partial z} \frac{\partial^{2} v}{\partial r \partial z}-\frac{\partial v}{\partial r} \frac{\partial^{2} v}{\partial z^{2}} \\
& \left.+u \frac{\partial^{3} u}{\partial r \partial z^{2}}+\frac{\partial u}{\partial z} \frac{\partial^{2} u}{\partial z \partial r}+w \frac{\partial^{3} u}{\partial z^{3}}+\frac{\partial w}{\partial z} \frac{\partial^{2} u}{\partial z^{2}}-\frac{1}{r}\left(\frac{\partial v}{\partial z}\right)^{2}\right] \\
& {\left[u \frac{\partial v}{\partial r}+\frac{u v}{r}+w \frac{\partial v}{\partial z}\right]=\frac{\partial^{2} v}{\partial z^{2}}-2 \Omega \delta^{2} v u+\frac{\alpha_{1} \Omega}{\mu}\left[\frac{2}{r} \frac{\partial v}{\partial z} \frac{\partial u}{\partial z}+\frac{\partial v}{\partial z} \frac{\partial^{2} u}{\partial r \partial z}+\frac{\partial v}{\partial z} \frac{\partial^{2} w}{\partial z^{2}}+\right.} \\
& \left.\frac{\partial w}{\partial z} \frac{\partial^{2} v}{\partial z^{2}}-\frac{u}{r} \frac{\partial^{2} v}{\partial z^{2}}+\frac{v}{r} \frac{\partial^{2} u}{\partial z^{2}}+u \frac{\partial^{3} v}{\partial z^{2} \partial r}+\frac{\partial u}{\partial z} \frac{\partial^{2} v}{\partial z \partial r}+w \frac{\partial^{3} v}{\partial z^{3}}+\frac{\partial w}{\partial z} \frac{\partial^{2} v}{\partial z^{2}}\right] \\
& -2 \frac{\partial u}{\partial z} \frac{\partial^{2} u}{\partial z^{2}}-2 \frac{\partial v}{\partial z} \frac{\partial^{2} v}{\partial z^{2}}=0
\end{aligned}
$$

The terms $2 \Omega \delta^{2} v$ and $2 \rho \Omega \delta^{2} v v^{2}$ are the projections of the Coriolis onto the axis $r$ and $\theta$, respectively, while the term $\rho \Omega \delta^{2} v r$ is the projection of centrifugal force. where $f(z)$ and $g(z)$ can be considered as dimensionless velocities onto the $\mathrm{r}$-axis depends only on $\mathrm{z}$.

Boundary conditions in non-dimensional form are

$u \quad=\gamma r ; \quad v=r ; \quad w=0 \quad$ atz $=0$

$u \quad \rightarrow 0 ; \quad v \rightarrow 0 \quad$ asz $\rightarrow \infty$

where $\gamma=\frac{A}{\Omega}$ is the stretching parameter.

To convert the partial differential equation into ordinary differential equation we make the following transformations.

$$
u=-\frac{r}{2} f^{\prime}(z), \quad v=r g(z), \quad w=f(z)
$$

The continuity equation satisfied identically and momentum equations take the following form after algebraic manipulation

$$
\begin{aligned}
& f^{\prime 2}-2 f f^{\prime \prime}-4 g^{2}+2 f^{\prime \prime \prime}-4 \beta[1+2 g]+\alpha\left[4 f^{\prime f^{\prime \prime \prime}}+8 g^{2}+4 g g^{\prime \prime}-f^{\prime 2}+2 f f^{(i)}\right]=0 \\
& f^{\prime} g-f g^{\prime}+g^{\prime \prime}+\beta f^{\prime}+\alpha\left[-g^{\prime} f^{\prime \prime}+2 g^{\prime \prime} f^{\prime}-g f^{\prime \prime \prime}+f g^{\prime \prime \prime}\right]=0 \\
& f^{\prime \prime} f^{\prime \prime \prime}+4 g^{\prime} g^{\prime \prime}=0
\end{aligned}
$$

Boundary conditions becomes

$$
f^{\prime}(0)=-2 \gamma, \quad f(0)=0, \quad g(0)=1, \quad f^{\prime}(\infty)=0, \quad g(\infty)=0
$$

where $\alpha=\frac{\alpha_{1} \Omega}{\mu}$ is viscoellastic parameter, $\beta=\Omega \delta^{2} v$ is the rotational number, $E_{k}=\frac{v}{\Omega S^{2}}$ is Ekman number and $\mathrm{S}$ is the height.

The model applies strictly to an infinite disk, but can be applied to a finite disk of radius $\mathrm{R}$, provided that $\mathrm{R}>\delta$ is satisfied.

\section{Solution by Homotopy Analysis Method}

By the HAM method, the functions $f(z)$ and $g(z)$ as:

$$
\begin{aligned}
& f_{m}(z)=\sum_{n=0}^{\infty}, \sum_{n=0}^{\infty} \alpha_{m, n}^{k} z^{k} \exp (-n z), \\
& g_{m}(z)=\sum_{n=0}^{\infty} \sum_{n=0}^{\infty} b_{m, n}^{k} z^{k} \exp (-n z)
\end{aligned}
$$

where $a_{m, n}^{k} z^{k}$ and $b_{m, n}^{k} z^{k}$ are the coefficients to be determined. Initial guess and auxiliary linear operator are chosen as follows:

$$
\begin{aligned}
& f_{0}(z)=\gamma\left(-1+e^{z}\right) e^{-z}, \quad g_{0}(z)=e^{-\frac{z}{2}} \\
& L_{f}=\frac{\partial^{3} f}{\partial z^{3}}-\frac{\partial f}{\partial z}, \quad L_{g}=2 \frac{\partial^{2} g}{\partial z^{2}}+\frac{\partial g}{\partial z},
\end{aligned}
$$

the above auxiliary linear operators have the following properties

$$
\begin{array}{ll}
L_{f}\left(c_{1}+c_{2} e^{z}+c_{3} e^{-z}\right) & =0, \\
L_{g}\left(c_{4}+c_{5} e^{-z / 2}\right) & =0,
\end{array}
$$

where $c_{i}(i=1-5)$ are arbitrary constants. The zeroth order deformation problems can be obtain as:

$$
\begin{aligned}
& (1-q) L_{f}\left[\hat{f}(z ; q)-f_{0}(z)\right]=q h_{f} N_{f}[\hat{f}(z ; q)] . \\
& (1-q) L_{g}\left[\hat{g}(z ; q)-g_{0}(z)\right]=q h_{g} N_{g}[\hat{f}(z ; q), \hat{g}(z ; q)], \\
& N[\hat{f}(z ; q)]=\left(\frac{\partial \hat{f}(z ; q)}{\partial z}\right)^{2}\left(\frac{\partial^{3} \hat{f}(z ; q)}{\partial z^{3}}\right)^{2}+8 \hat{f}(z ; q) \frac{\partial \hat{g}(z ; q)}{\partial z} \frac{\partial^{2} \hat{g}(z ; q)}{\partial z^{2}} \frac{\partial^{3} \hat{f}(z ; q)}{\partial z^{3}} \\
& -4(\hat{g}(z ; q))^{2}\left(\frac{\partial^{3} \hat{f}(z ; q)}{\partial z^{3}}\right)^{2}+2\left(\frac{\partial^{3} \hat{f}(z ; q)}{\partial z^{3}}\right)^{3}-4(1+2 \hat{g}(z ; q))\left(\frac{\partial^{3} \hat{f}(z ; q)}{\partial z^{3}}\right)^{2} \\
& +4\left[\frac{\partial \hat{f}(z ; q)}{\partial z}\left(\frac{\partial^{3} \hat{f}(z ; q)}{\partial z^{3}}\right)^{3}+2\left(\frac{\partial \hat{g}(z ; q)}{\partial z}\right)^{2}\left(\frac{\partial^{3} \hat{f}(z ; q)}{\partial z^{3}}\right)^{2}\right. \\
& \hat{g}(z q) \frac{\partial \hat{g}(z q)}{\partial}\left(\frac{\partial^{3} \hat{f}(z ; q)}{\partial z^{3}}\right)^{2}+4\left(\frac{\partial \hat{g}(z ; q)}{\partial z}\right)^{2}\left(\frac{\partial^{2} \hat{g}(z ; q)}{\partial z^{2}}\right)^{2}+\hat{f}(z q) \frac{\partial^{4} \hat{f}(z ; q)}{\partial z^{4}}\left(\frac{\partial^{3} \hat{f}(z ; q)}{\partial z^{3}} 2\right] \\
& \left.N_{\varepsilon}[\hat{f}(z ; q), \hat{g}(z ; q)]=\hat{g}(z ; q)\right) \frac{\partial \hat{f}(z ; q)}{\partial z} \frac{\partial^{3} \hat{f}(z ; q)}{\partial z^{3}}-\hat{f}(z ; q) \frac{\partial \hat{g}(z ; q)}{\partial z} \frac{\partial^{3} \hat{f}(z ; q)}{\partial z^{3}} \\
& +\frac{\partial^{2} \hat{g}(z ; q)}{\partial z^{2}} \frac{\partial^{3} \hat{f}(z ; q)}{\partial z^{3}}+\beta \frac{\partial \hat{f}(z ; q)}{\partial z} \frac{\partial^{3} f(z ; q)}{\partial z^{3}} \\
& +\alpha\left[4\left(\frac{\partial \hat{g}(z ; q)}{\partial z}\right)^{\frac{\partial^{2}}{\partial z} \hat{g}(z ; q)} \frac{\partial z}{\partial z^{2}}+2 \frac{\partial^{2} \hat{g}(z ; q)}{\partial z^{2}} \frac{\partial \hat{f}(z ; q)}{\partial z} \frac{\partial^{3} \hat{f}(z ; q)}{\partial z^{3}}\right. \\
& \left.+2 \hat{f}(z ; q) \frac{\partial^{3} \hat{g}(z ; q)}{\partial z^{3}} \frac{\partial^{3} \hat{f}(z ; q)}{\partial z^{3}}-\hat{g}(z ; q)\left(\frac{\partial^{3} \hat{f}(z ; q)}{\partial z^{3}}\right)^{2}+\hat{f}(z ; q) \frac{\partial^{3} \hat{g}(z ; q)}{\partial z^{3}} \frac{\partial^{3} \hat{f}(z ; q)}{\partial z^{3}}\right],
\end{aligned}
$$

where $q$ is an embedding parameter, $h_{f}$ and $h_{g}$ are the non-zero auxiliary parameter and $N_{f}$ and $N_{\hat{a} \in \dot{c}}$ are nonlinear operators. 
For $\mathrm{q}=0$ and $\mathrm{q}=1$ we have:

$$
\begin{array}{ll}
\hat{f}(z ; 0)=f_{0}(z), & \hat{f}(z ; 1)=f(z) \\
\hat{g}(z ; 0)=g_{0}(z), & \hat{g}(z ; 1)=g(z) .
\end{array}
$$

Therefore, as the embedding parameter $q$ increases from 0 to 1 , $\hat{f}(z ; q)$ and $\hat{g}(z ; q)$ varies from their initial guesses $f_{0}$ and $g_{0}$ to the exact solutions $f(z)$ and $g(z)$ respectively. Taylor's series expansion of these functions yields:

$$
\begin{aligned}
& f(z q)=f_{0}(z)+\sum_{m=1}^{\infty} f_{m}(z) q^{m}, \\
& g(z q)=g_{0}(z)+\sum_{m=1}^{\infty} g_{m}(z) q^{m},
\end{aligned}
$$

where

$$
f_{m}=\left.\frac{1}{m !} \frac{\partial^{m} f(z ; q)}{\partial z^{m}}\right|_{q=0}, \quad g_{m}=\left.\frac{1}{m !} \frac{\partial^{m} g(z ; q)}{\partial z^{m}}\right|_{q=0} .
$$

Keeping in mind the above series depends on $h_{f}$ and $h_{g}$ On the assumption that the non-zero auxiliary parameters are chosen so that Eq. (39) converge at $q=1$.

Therefore we can obtain:

$$
\begin{aligned}
& f(z)=f_{0}(z)+\sum_{m=1}^{\infty} f_{m}(z), \\
& g(z)=g_{0}(z)+\sum_{m=1}^{\infty} g_{m}(z) .
\end{aligned}
$$

Differentiating $\mathrm{m}$-times the zeroth order deformation equations (35) and (36)one has the mth order deformation equations as:

$$
\begin{aligned}
& L_{f}\left[f_{m}(z)-\chi_{m} f_{m-1}(z)\right]=h_{f} R_{f, m}(z), \\
& L_{g}\left[g_{m}(z)-\chi_{m} g_{m-1}(z)\right]=h_{g} R_{g, m}(z),
\end{aligned}
$$

where, the boundary conditions (29) takes the form

$$
\begin{aligned}
& f_{m}(0)=f_{m}^{\prime}(0)=f_{m}^{\prime}(\infty)=0, \\
& g_{m}(0)=g_{m}(\infty)=0, \\
& R_{f, m}(z)=\sum_{j=0}^{m-3} \frac{\partial f_{j}(z)}{\partial z} \frac{\partial f_{j-m-1}(z)}{\partial z} \frac{\partial^{3} f_{j-m-2}(z)}{\partial z^{3}} \frac{\partial^{3} f_{j-m-3}(z)}{\partial z^{3}} \\
& +8 \sum_{j=0}^{m-3} f_{j}(z) \frac{\partial g_{j-m-1}(z)}{\partial z} \frac{\partial^{2} g_{j-m-2}(z)}{\partial z^{2}} \frac{\partial^{3} f_{j-m-3}(z)}{\partial z^{3}} \\
& -4 \sum_{j=0}^{m-3} g_{j}(z) g_{j-m-1}(z) \frac{\partial^{3} f_{j-m-2}(z)}{\partial z^{3}} \frac{\partial^{3} f_{j-m-3}(z)}{\partial z^{3}} \\
& +2 \sum_{j=0}^{m-2} \frac{\partial^{3} f_{j}(z)}{\partial^{3} z} \frac{\partial^{3} f_{j-m-1}(z)}{\partial z^{3}} \frac{\partial^{3} f_{j-m-2}(z)}{\partial z^{3}} \\
& -4 \beta\left[\sum_{j=0}^{m-2} \frac{\partial^{3} f_{j}(z)}{\partial^{3} z} \frac{\partial^{3} f_{j-m-1}(z)}{\partial z^{3}}\right. \\
& \left.+2 \sum_{j=0}^{m-2} g_{j}(z) \frac{\partial^{3} f_{j-m-1}(z)}{\partial z^{3}} \frac{\partial^{3} f_{j-m-2}(z)}{\partial z^{3}}\right] \\
& +\alpha\left[4 \sum_{j=0}^{m-3} \frac{\partial f_{j}(z)}{\partial z} \frac{\partial^{3} f_{j-m-1}(z)}{\partial z^{3}} \frac{\partial^{3} f_{j-m-2}(z)}{\partial z^{3}} \frac{\partial^{3} f_{j-m-3}(z)}{\partial z^{3}}\right. \\
& +8 \sum_{j=0}^{m-3} \frac{\partial g_{j}(z)}{\partial z} \frac{\partial g_{j-m-1}(z)}{\partial z} \frac{\partial^{3} f_{j-m-2}(z)}{\partial z^{3}} \frac{\partial^{3} f_{j-m-3}(z)}{\partial z^{3}} \\
& +4 \sum_{j=0}^{m-3} g_{j}(z) \frac{\partial^{2} g_{j-m-1}(z)}{\partial z^{2}} \frac{\partial^{3} f_{j-m-2}(z)}{\partial z^{3}} \frac{\partial^{3} f_{j-m-3}(z)}{\partial z^{3}} \\
& +16 \sum_{j=0}^{m-3} \frac{\partial g_{j}(z)}{\partial z} \frac{\partial g_{j-m-1}(z)}{\partial z} \frac{\partial^{2} g_{j-m-2}(z)}{\partial z^{2}} \frac{\partial^{2} g_{j-m-3}(z)}{\partial z^{2}} \\
& \left.+\sum_{j=0}^{m-3} f_{j}(z) \frac{\partial^{4} f_{j-m-1}(z)}{\partial z^{4}} \frac{\partial^{3} f_{j-m-2}(z)}{\partial z^{3}} \frac{\partial^{3} f_{j-m-3}(z)}{\partial z^{3}}\right], \\
&
\end{aligned}
$$

$$
\begin{aligned}
& R_{g, m}(z)==\sum_{j=0}^{m-2} g_{j}(z) \frac{\partial f_{j-m-1}(z)}{\partial z} \frac{\partial^{3} f_{j-m-2}(z)}{\partial z^{3}}-\sum_{j=0}^{m-2} f_{j}(z) \frac{\partial g_{j-m-1}(z)}{\partial z} \frac{\partial^{3} f_{j-m-2}(z)}{\partial z^{3}} \\
& +\sum_{j=0}^{m-1} \frac{\partial^{2} g_{j}(z)}{\partial z^{2}} \frac{\partial^{3} f_{j-m-1}(z)}{\partial z^{3}}+\beta \sum_{j=0}^{m-1} \frac{\partial f_{j}(z)}{\partial z} \frac{\partial^{3} f_{j-m-1}(z)}{\partial z^{3}} \\
& +\alpha\left[4 \sum_{j=0}^{m-2} \frac{\partial g_{j}(z)}{\partial z} \frac{\partial g_{j-m-1}(z)}{\partial z} \frac{\partial^{2} g_{j-m-2}(z)}{\partial z^{2}}\right. \\
& +2 \sum_{j=0}^{m-2} \frac{\partial^{2} g_{j}(z)}{\partial z^{2}} \frac{\partial f_{j-m-1}(z)}{\partial z} \frac{\partial^{3} f_{j-m-2}(z)}{\partial z^{3}}+2 \sum_{j=0}^{m-2} f_{j}(z) \frac{\partial^{3} g_{j-m-1}(z)}{\partial z^{3}} \frac{\partial^{3} f_{j-m-2}(z)}{\partial z^{3}} \\
& \left.-\sum_{j=0}^{m-2} g_{j}(z) \frac{\partial^{3} f_{j-m-1}(z)}{\partial z^{3}} \frac{\partial^{3} f_{j-m-2}(z)}{\partial z^{3}}+\sum_{j=0}^{m-2} f_{j}(z) \frac{\partial^{3} g_{j-m-1}(z)}{\partial z^{3}} \frac{\partial^{3} f_{j-m-2}(z)}{\partial z^{3}}\right] \\
& \chi_{m}=\left(\begin{array}{ll}
1 & \mathrm{~m}>1, \\
0 & \mathrm{~m} \leq 1
\end{array}\right.
\end{aligned}
$$

Finally, the general solution may be written as follows:

$$
\begin{aligned}
& f_{m}(z)=f_{m}^{*}+c_{1}+c_{2} e^{z}+c_{3} e^{-z} \\
& g_{m}(z)=g_{m}^{*}+c_{4}+c_{5} e^{\frac{-z}{2}}
\end{aligned}
$$

Where $f_{m}^{*}$ and $g_{m}^{*}$ are the special solutions.

\section{Error Analysis}

To perform analysis of the problem under consideration we made the first error analysis to make sure that our analysis are reliable upto the scale of minimum residual error. Before to discus and give physical predictions we perform error analysis to investigate the validity of the HAM techniques. For this purpose, Figure 2 and Tables 1 and 2 are made. Table 1 represents the nonzero auxiliary convergence control parameters $h_{f}$ and $h_{g}$ and the minimum values of total averaged squared residual errors executed for different orders of approximations. the total squared residual error $\varepsilon_{m}^{t}$ can be minimized by increasing the order of approximations. Here, it can be seen that increasing the order of approximations the total squared residual errors are reduced. Table 2 illustrate the individual average squared residual error at different orders of approximations. Besides this Figure 2 also shows the maximum average squared residual error at different orders of approximation. It

\begin{tabular}{|c|c|c|c|}
\hline $\begin{array}{c}\text { Order of } \\
\text { approximation }\end{array}$ & $\boldsymbol{h}_{\boldsymbol{f}}$ & $\boldsymbol{h}_{\boldsymbol{g}}$ & $\boldsymbol{\varepsilon}_{\boldsymbol{m}}^{\boldsymbol{t}}$ \\
\hline 0 & 0 & 0 & $8.75827 \times 10^{-4}$ \\
\hline & -0.0692346 & -25.5265 & $2.61041 \times 10^{-4}$ \\
\hline & -0.0823316 & -39.7366 & $1.15004 \times 10^{-5}$ \\
\hline & -0.0854184 & -28.1879 & $6.57551 \times 10^{-6}$ \\
\hline & -0.103503 & -33.9341 & $3.73624 \times 10^{-6}$ \\
\hline & -0.0954044 & -23.8306 & $2.83147 \times 10^{-6}$ \\
\hline
\end{tabular}

Table 1: Optimal values of convergence control parameters versus different orders of approximation.

\begin{tabular}{|l|c|c|c|}
\hline $\boldsymbol{m}$ & $\boldsymbol{\varepsilon}_{\boldsymbol{m}}^{f}$ & $\boldsymbol{\varepsilon}_{\boldsymbol{m}}^{g}$ & Used CPU time \\
\hline 2 & $3.36317 \times 10^{-5}$ & $2.6919 \times 10^{-6}$ & 2.46874 (seconds) \\
\hline & $6.98761 \times 10^{-6}$ & $2.54788 \times 10^{-6}$ & 11.0518 (seconds) \\
\hline & $3.50986 \times 10^{-6}$ & $2.49417 \times 10^{-6}$ & 34.3474 (seconds) \\
\hline & $2.21929 \times 10^{-6}$ & $2.45682 \times 10^{-6}$ & 80.3647 (seconds) \\
\hline & $1.5526 \times 10^{-6}$ & $2.4233 \times 10^{-6}$ & 171.906 (seconds) \\
\hline & $1.15881 \times 10^{-6}$ & $2.39127 \times 10^{-6}$ & 206.126 (seconds) \\
\hline & $9.05047 \times 10^{-7}$ & $2.3602 \times 10^{-6}$ & 632.98 (seconds) \\
\hline & $7.30949 \times 10^{-7}$ & $2.32987 \times 10^{-6}$ & 1298.4 (seconds) \\
\hline & $6.05727 \times 10^{-7}$ & $2.3002 \times 10^{-6}$ & 2435.04 (seconds) \\
\hline & $5.12274 \times 10^{-7}$ & $2.27111 \times 10^{-6}$ & 4670.28 (seconds) \\
\hline
\end{tabular}

Table 2: Individual averaged squared residual errors using optimal values of auxiliary parameters. Using $\gamma=0.01 ; \alpha=0.1 ; \beta=0.5$. 

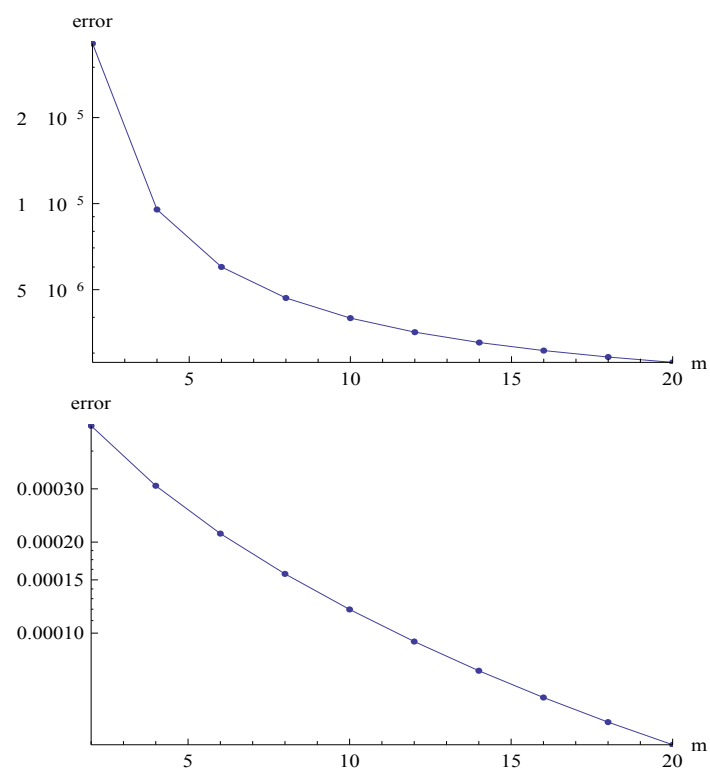

Figure 2: Residual error profile for the axial component of velocity versus order of approximation by taking fixed values of $\alpha, \beta$ and $\gamma$

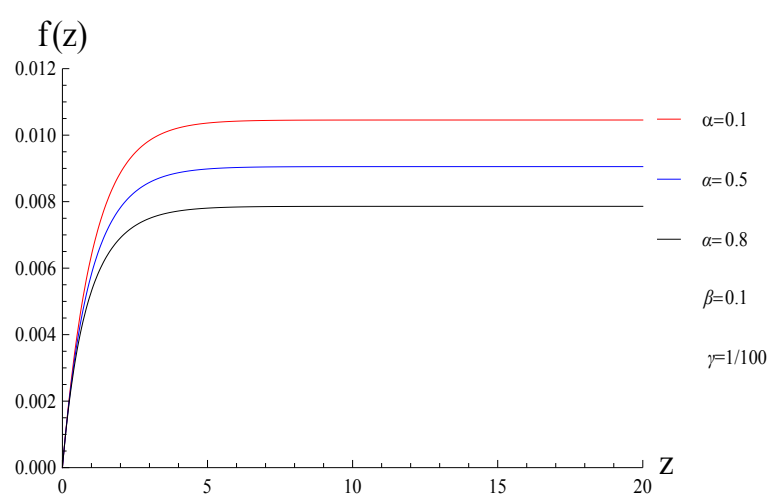

Figure 3: Axial component of velocity profile $f(z)$ for various values of viscoelastic parameter $\alpha$, for fixed values of $\beta=0.1$ and $\gamma=0.01$.

can also be observed that the total averaged squared errors and average squared residual errors are decreasing as the order of approximation is increasing for different values of physical parameters $\alpha$ and $\beta$.

\section{Results and Discussion}

In this section, we present graphical results of the system of coupled nonlinear ODE's given in eqn. (26) and eqn. (27) corresponding the boundary conditions (28). Numerical Solution is obtained by means of the BVPh2.0, a HAM Mathematica package $[9,10]$. For better analysis, Figures 2-16 are plotted. In order to get the numerical solutions of the above equations, it was translated into BVPh 2.0 program in Mathemetica by setting the required error $10^{-10}$. The semi infinite domain $\mathrm{z} \varepsilon[0, \infty)$ is replaced by a finite domain $\mathrm{z} \varepsilon\left[0, \mathrm{z}_{\infty}\right]$. In practice, $\mathrm{z}_{\infty}$ should be chosen sufficiently large so that the numerical solution closely approximates the terminal boundary conditions.

Figures 3-5 show the influence of the viscoelastic parameter $\alpha$ on the non dimensional axial velocity component $f(z)$ for fixed values of $\gamma=0.01$ and $\beta=0.1,0.5,5$, respectively. It is observed that increasing $\alpha$ the speed of the flow reduces. This is due to the fact, that increasing non-Newtonian effect $\alpha$ shear forces increases in the fluid domain
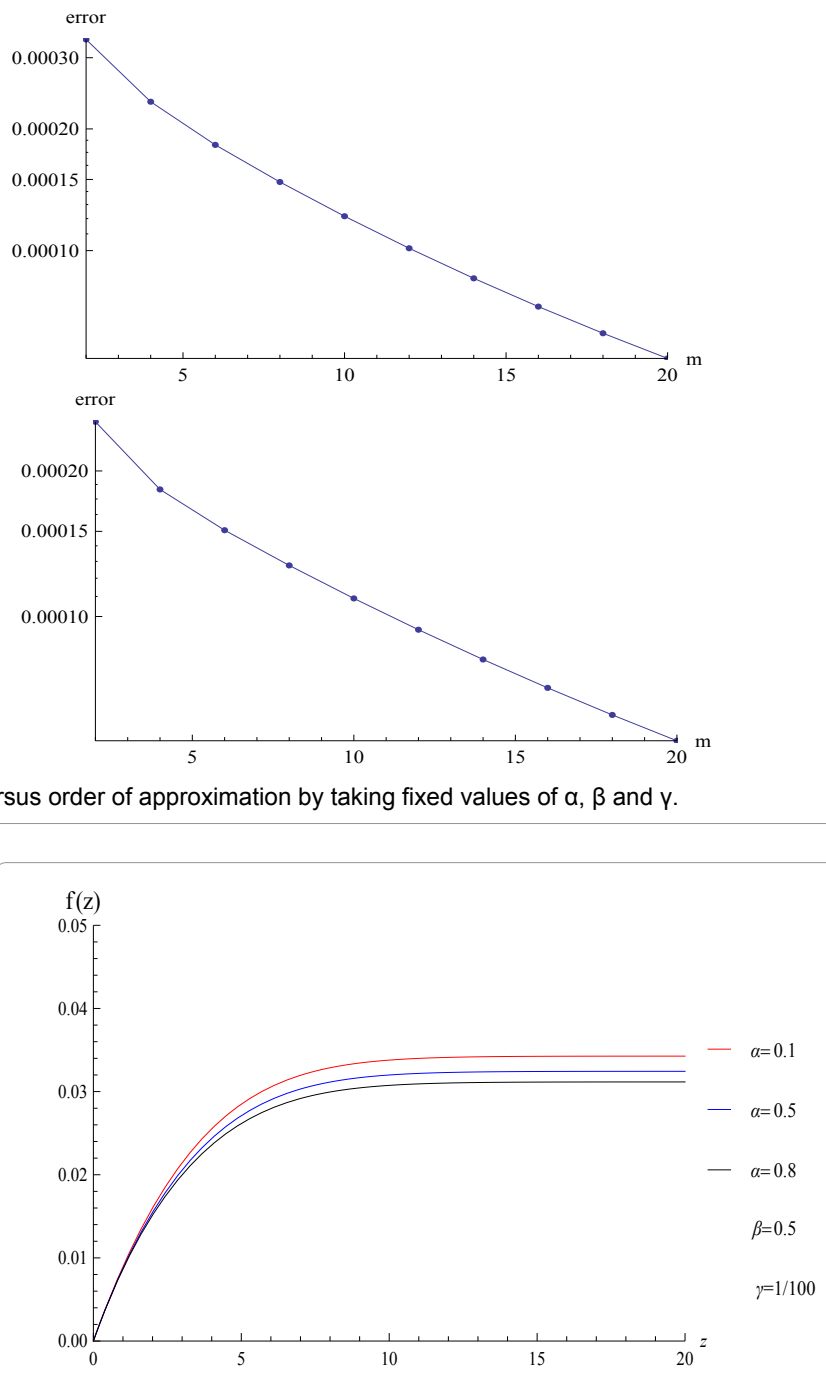

Figure 4: Axial component of velocity profile $f(z)$ for various values of viscoelastic parameter $\alpha$, for fixed values of $\beta=0.5$ and $\gamma=0.01$.

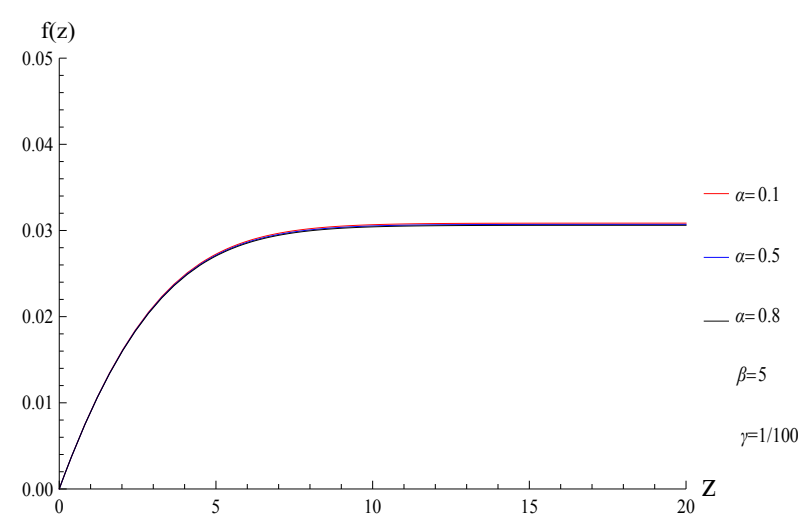

Figure 5: Axial component of velocity profile $f(z)$ for various values of viscoelastic parameter $\alpha$, for fixed values of $\beta=0.5$ and $\gamma=0.01$.

which reduced the speed of flow. Also it can be noticed that large values of rotation number $\beta$ dominate the influence of viscoelastic parameter $\alpha$, while the effect of $\alpha$, can be seen only for small values of $\beta$ [11-15]. 


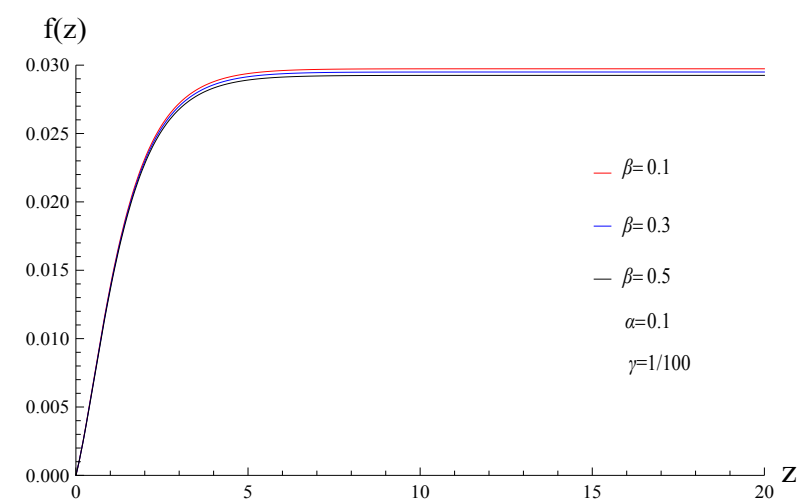

Figure 6: Axial component of velocity profile $f(z)$ for various values of rotation parameter $\beta$, for fixed values of $\alpha=0.1$ and $\gamma=0.01$.

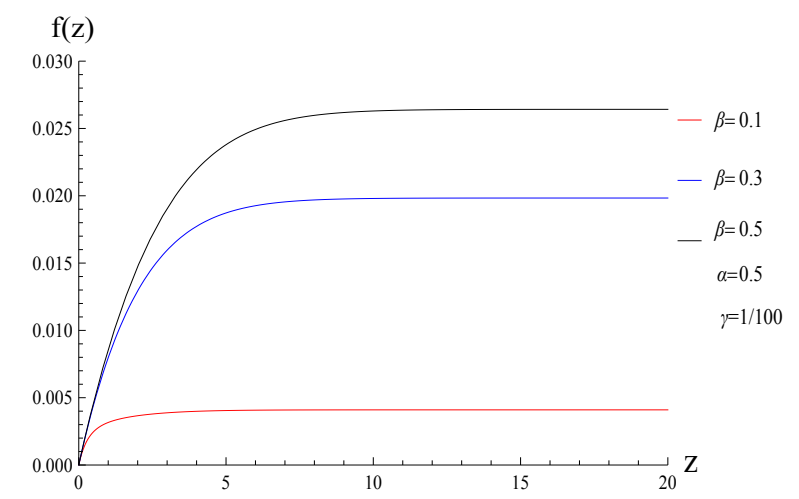

Figure 7: Axial component of velocity profile $f(z)$ for various values of rotation parameter $\beta$, for fixed values of $\alpha=0.5$ and $\gamma=0.01$.

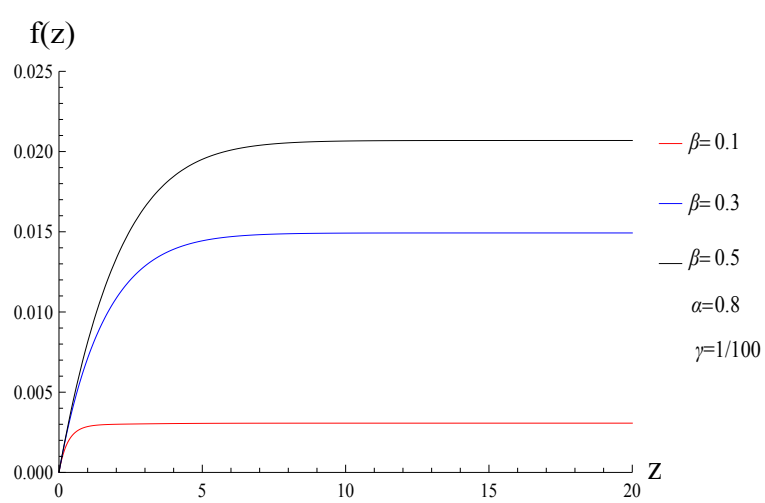

Figure 8: Axial component of velocity profile $f(z)$ for various values of rotation parameter $\beta$, for fixed values of $\alpha=0.8$ and $\gamma=0.01$.

The variation of dimensionless axial velocity component $f(z)$ versus axial direction $z$ for different values of rotation number $\beta$ are plotted in Figs. 6-8, for fixed values of slip parameter $\gamma=0.1,0.5$ and $\gamma=0.8$, respectively. It can be seen that increasing the value of $\beta$ results in an increase in the axial velocity component near the disk; however, as expected the rate of increase of the axial velocity is negligible far away from the disk.

To investigate the fluid velocity along azimuthal direction with and without Coriolis and centrifugal force Figure 9 is made. Near the disk there is no effect on the velocity component $f$ and far away these forces $\mathrm{f}(\mathrm{z})$

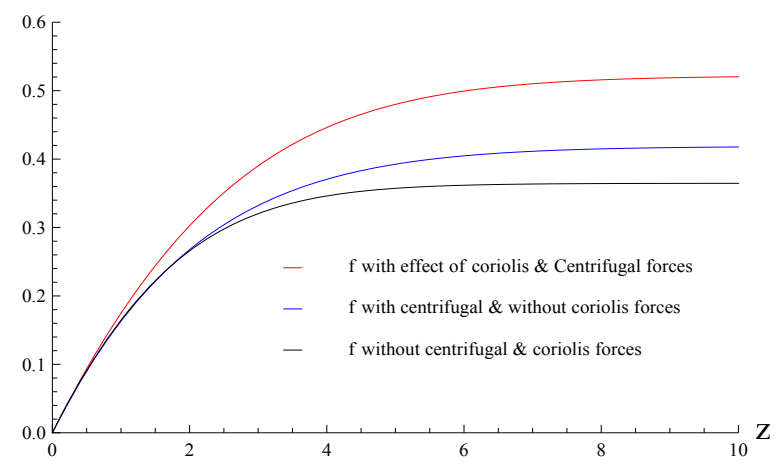

Figure 9: Axial component of velocity profile $f(z)$ for various values of rotation parameter $\beta$, for fixed values of $\alpha=0.8$ and $\gamma=0.01$.

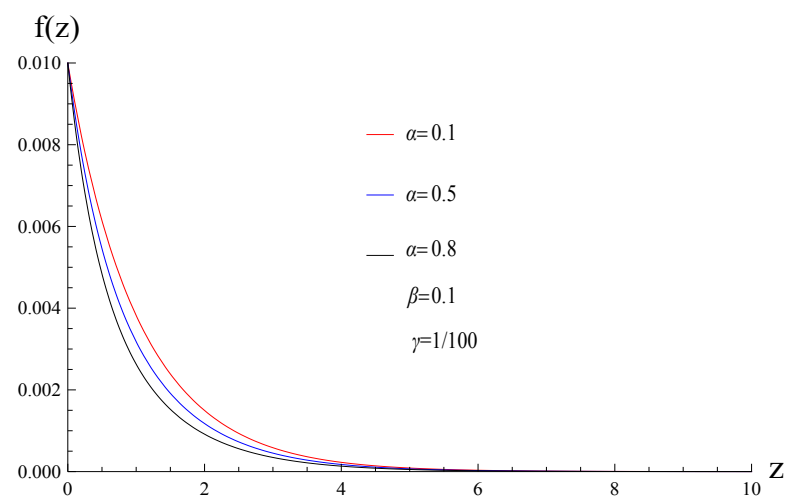

Figure 10: Radial component of velocity profile $f(z)$ for various values of rotation parameter $\alpha$, for fixed values of $\beta=0.1$ and $\gamma=0.01$.

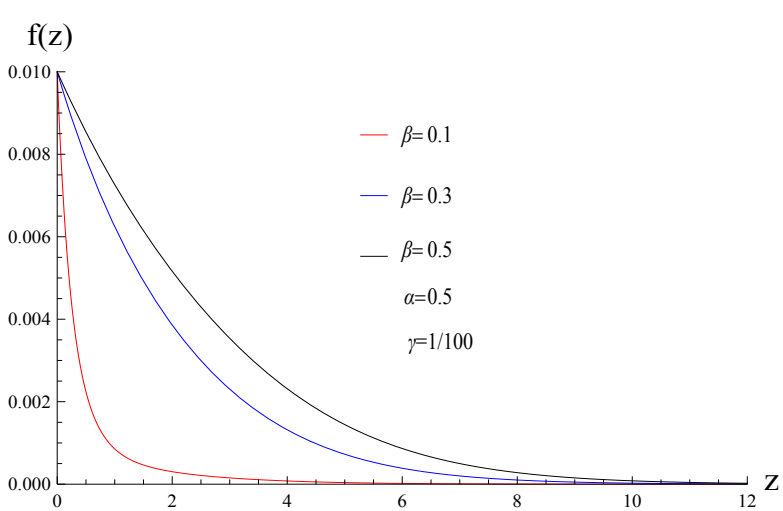

Figure 11: Radial component of velocity profile $f^{\prime}(z)$ for various values of rotation parameter $\beta$, for fixed values of $\alpha=0.5$ and $\gamma=0.01$.

effect $f(z)$, the combine effect of these forces clearly effect the velocity field $f$. As expected, near the disk the Coriolis and centrifugal forces balance the effect of each other [16-25].

Figures 10-12 depict the effect of viscoelastic parameter $\alpha$ rotation parameter $\beta$ and slip parameter $\gamma$ for selected values of rotation number $\beta$ and slip parameter $\gamma$. One can see that increasing nonNewtonian effect the boundary layer region decreases and increasing rotation number the boundary layer increases and similar case is seen for increasing slip parameter $\gamma$. The influence of centrifugal and Coriolis forces on radial velocity component are graphed in Figure 13. 


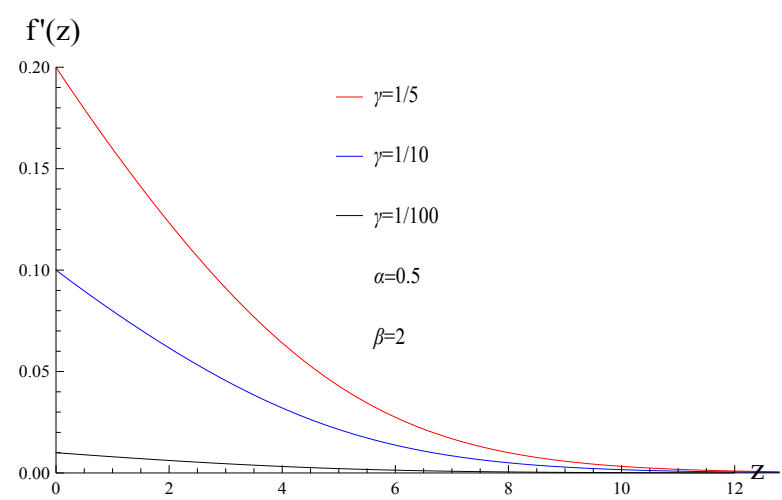

Figure 12: Radial component of velocity profile $f^{\prime}(z)$ for various values of rotation parameter $\gamma$, for fixed values of $\alpha=0.5$ and $\beta=2$.

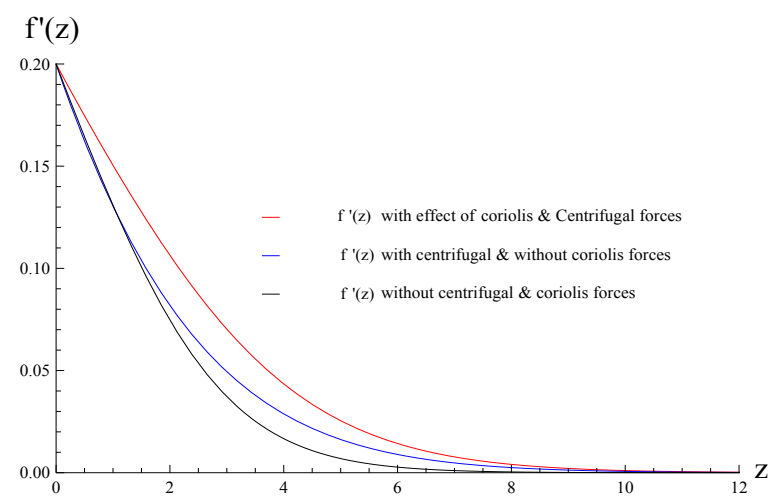

Figure 13: Radial component of velocity profile $f^{\prime}(z)$ for various values of rotation parameter $\gamma$, for fixed values of $\alpha=0.5$ and $\beta=2$.

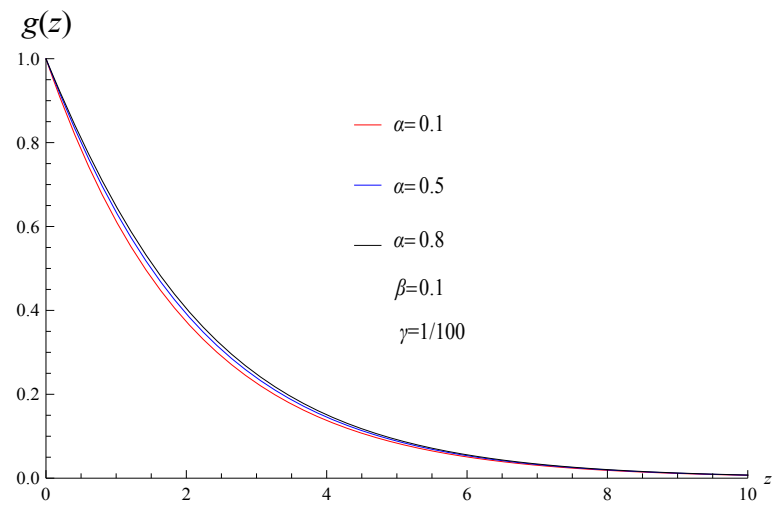

Figure 14: Azimuthal component of velocity profile $\mathrm{g}(\mathrm{z})$ for various values of viscoelastic parameter $\alpha$, for fixed values of $\beta=0.5$ and $y=0.01$.

It is interesting to note that the radial velocity increases by taking the effect of these forces. The effect of centrifugal force near the surface of the disk is seems to be negligible and far away from the surface of the rotating disk its effect can be seen clearly. The influence of different values of viscoelastic parameter $\alpha=0.10 .5,0.8$ for fixed values of $\beta=0.5$ and $\gamma=0.01$ on radial velocity component are plotted in Figure 14 . It is also interesting to note that increasing the parameter $\alpha$ the azimuthal velocity component also increases but for small values of $\beta$ and $\gamma$ this increase is small in magnitude [26-32].

Table 3 and 4 are made to observed the variation of azimuthal

\begin{tabular}{|c|c|c|c|}
\hline $\mathbf{z}$ & $\boldsymbol{\alpha = 0 . 1}$ & $\boldsymbol{\alpha = 0 . 5}$ & $\boldsymbol{\alpha = 0 . 8}$ \\
\hline 0.0 & 1 & 1 & 1 \\
\hline .0 & 0.607597 & 0.611658 & 0.614624 \\
\hline .0 & 0.368775 & 0.37217 & 0.374672 \\
\hline .0 & 0.223733 & 0.226002 & 0.22768 \\
\hline .0 & 0.135716 & 0.137139 & 0.138193 \\
\hline .0 & 0.0823197 & 0.0831935 & 0.0838407 \\
\hline .0 & 0.0499304 & 0.0504628 & 0.0508571 \\
\hline .0 & 0.0302846 & 0.030608 & 0.0308476 \\
\hline .0 & 0.0183686 & 0.0185649 & 0.0187103 \\
\hline .0 & 0.0111412 & 0.0112602 & 0.0113484 \\
\hline .0 & 0.00675747 & 0.0068297 & 0.0068832 \\
\hline
\end{tabular}

Table 3: Axial component velocity profile $g(z)$ for different values of $\alpha$. Using $\beta=2$; $y=0.01$.

\begin{tabular}{|c|c|c|c|}
\hline $\mathbf{z}$ & $\mathbf{y = 0 . 2 0}$ & $\mathbf{y = 0 ~ . 1}$ & $\mathbf{~ = 0 . 0 1}$ \\
\hline 0.0 & 1 & 1 & 1 \\
\hline .0 & 0.61381 & 0.6127 & 0.612154 \\
\hline .0 & 0.374467 & 0.373206 & 0.372588 \\
\hline .0 & 0.227821 & 0.226789 & 0.226282 \\
\hline .0 & 0.138405 & 0.137676 & 0.137315 \\
\hline .0 & 0.0840168 & 0.0835399 & 0.0833018 \\
\hline .0 & 0.0509798 & 0.0506798 & 0.0505289 \\
\hline .0 & 0.0309269 & 0.0307418 & 0.0306482 \\
\hline .0 & 0.0187598 & 0.0186467 & 0.0185893 \\
\hline .0 & 0.0113789 & 0.0113101 & 0.011275 \\
\hline .0 & 0.00690179 & 0.00685998 & 0.00683867 \\
\hline
\end{tabular}

Table 4: Axial component velocity profile $g(z)$ for different values of $y$ using $\alpha=0.5$; $\beta=2$.

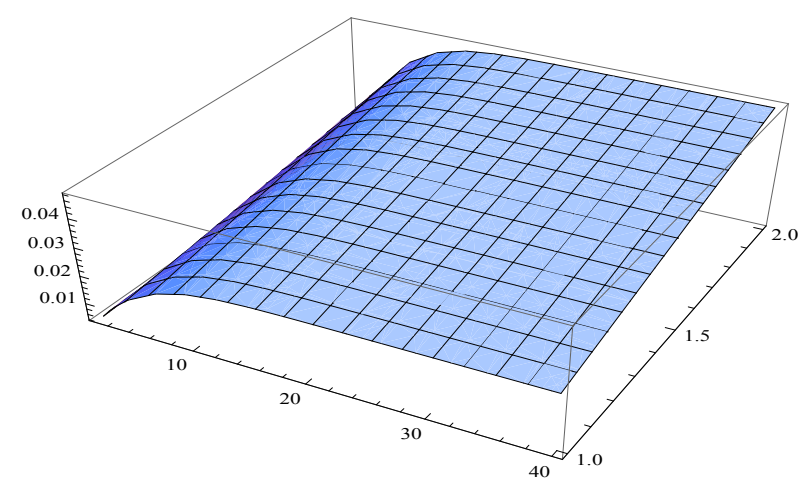

Figure 15: Three dimensional skich of the radial velocity component for the fixed values of $\alpha=0.1, \beta=0.5$ and $\gamma=0.01$.

velocity component for selected values of $\alpha$ and $\gamma$ for the fixed values of other parameters of interest. Here it can be seen that on increasing $\alpha$ and $\gamma$ the azimuthal component of velocity $g(z)$ also increases. Three dimensional radial and azimuthal velocity components are plotted in Figures 15 and 16 for different values of $\alpha=0.1, \beta=0.5$ and $\gamma=0.01$.

\section{Conclusion}

Three dimensional rotating flow over a long disk of a viscoelostic fluid under the influence of Coriolis and centrifugal forces are studied. From the context of transformations and dimensional analysis mathematical system of ODE's are obtained. A careful analysis of the flow is carried out by means of HAM Mathematica package. The following conclusions are made during analysis: 
Citation: Shuaiba M, Shaha RA, Khana A (2017) Study of Second Grade Fluid over a Rotating Disk with Coriolis and Centrifugal Forces. J Phys Math 8: 242. doi: 10.4172/2090-0902.1000242

Page 8 of 8

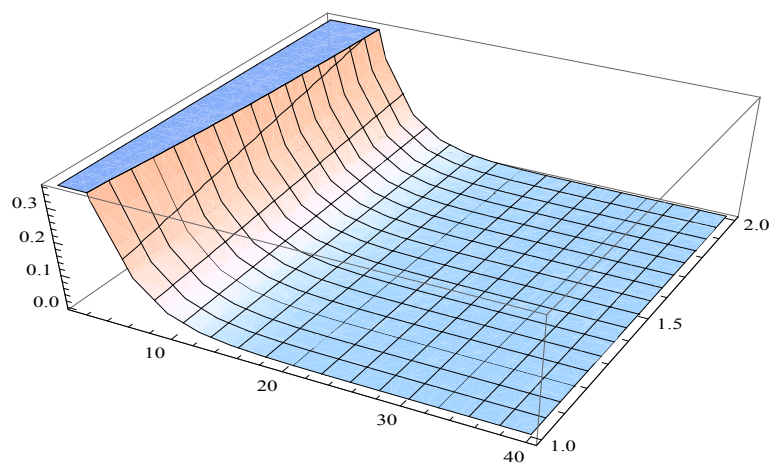

Figure 16: Three dimensional skich of the Axial velocity component for the fixed values of $\alpha=0.1, \beta=0.5$ and $y=0.01$.

1. It is concluded that increasing non-Newtonian parameter $\alpha$, the radial component and axial component of velocity increases, while the azimuthal component of velocity decreases.

2. It is also concluded that increasing the rotation number $\beta$, the radial and axial components of velocity profile also increases.

3. Further more, increasing slip effect causes the radial velocity component to increase.

4. Moreover, the radial and axial velocity component increase by taking the effect of centrifugal and Coriolis forces in the momentum equations. These finding abstruse and enrich our understanding about the boundary layer flow of second grade fluid.

\section{References}

1. Von Karman T (1921) Uber laminar and turbulent reibung. ZAMM 1: 233-235.

2. Cochran WG (1934) The flow due to a rotating disc. Proc Cambridge Philos Soc 30: 365-375

3. Benton ER (1966) On the flow due to a rotating disk. J Fluid Mech 24: 781-800.

4. Mithal KG (1961) On the effects of uniform high suction on the steady flow of non-Newtonian liquid due to a rotating disk. Quart J Mech App Math 14: 403410.

5. Attia HA (2003) Unsteady flow of non-Newtonian fluid above a rotating disk with heat transfer. International Journal of Heat and Mass Transfer 46: 2695-2700.

6. El-Mistikawy TMA, Attia HA (1991) The rotating disk flow in the presence of weak magnetic field. $4^{\text {th }}$ conference on theoretical and Applied mechanics, pp: 69-82.

7. Cham TS, Head MR (1969) Turbulent boundary-Layer flow on a rotating disk. J Fluid Mech 37: 129-147.

8. Mistikawy TMA, Attia HA, Megahed AA (1991) The rotating disk flow in the presence of weak magnetic field. Proc. $4^{\text {th }}$ Conference on Theoretical and Applied Mechanics, pp: 69-82.

9. Aboul Hassan AL, Attia HA (1997) The flow due to a rotating disk with Hall effect. Physics Letters A 228: 286-290.
10. Stuart JT (1954) On the effects of uniform suction on the steady flow due to a rotating disk. Quart. J Mech Appl Math 7: 446-457.

11. Kuiken HK (1971) The effect of normal blowing on the flow near a rotating disk of infinite extent. J Fluid Mech 47: 789-798.

12. Ockendon $H$ (1972) An asymptotic solution for steady flow above an infinite rotating disk with suction Quart. J Fluid Mech Appl Math 25: 291-301.

13. Attia HA (1998) Unsteady MHD flow near a rotating porous disk with uniform suction or injection. Fluid Dynamics Research 23: 283-290.

14. Attia HA, Aboul Hassan AL (2001) Effect of Hall current on the unsteady MHD flow due to a rotating disk with uniform suction or injection. Applied Mathematical Modelling 25: 1089-1098.

15. Attia HA (2002) The effectiveness of uniform suction injection on the unsteady flow due to a rotating disk with heat transfer. Int Comm Heat Mass Transf 29 653-661.

16. Erian FF, Tong YH ( 1971) Turbulent flow due to a rotating disk. Phys Fluids 14: 2588-2591.

17. Nayfeh AH (1971) Perturbation methods. Johan wiley and Sons, pp: 56-103

18. Erdogan ME (1976) Non-Newtonian flow due to non-coaxial rotations of a disk and a fluid at infinity. ZAMM, pp: 56-141.

19. Kohama Y (1984) Study on boundary layer transition of a rotating-disk. Acta Mech 50: 193-199.

20. Hall A (1986) An asymptotic investigation of the stationary modes of instability of the boundary layer on a rotating-disk. Proc Roy Soc London Ser A 406: 93-106.

21. Liao SJ (1992) propesd homotopy analysis techniques for the solution of nonlinear problem. shanghai Jioa Tong University.

22. Jarre S, Gal LE, Chauve MP (1996) Experimental study of rotating-disk instability. I Natural flow Phys Fluids 8: 496-508.

23. Aboul-Hassan AL, Attia HA (1997) The flow due to a rotating disk with Hall effect. Physics letters A 228: 286-290.

24. Siddiqui AM, Haroon T, Hayat T, Asgher S (2001) Unsteady MHD flow of a nonNewtonian fluid due to eccentric rotations of a porous disk and a fluid at infinity. Acta Mech 147: 99-109.

25. Polyanin AD (2001) Exact solutions to the Navier-Stokes equations with generalized separation of variables. Dokl Phys 46: 726-731.

26. Zhao Y, Lioa S (2002) User Guide to BVPh 2.0. Shanghai Jioa Tong University China, pp: 1-40.

27. Hayat T, Haroon T, Asghar S, Siddiqui AM (2003) MHD flow of a third grade fluid due to eccentric rotations of a porous disk and a fluid at infinity. Int J Nonlinear Mech 38: 501-511.

28. Liao SJ (2003) On the analytic solution of magneto hydrodynamic flows of nonNewtonian fluids over a stretching sheet. J Fluid Mech 488: 189-212.

29. Sajid M, Z lqbal, T Hayat, S Obaidat (2011) series solution for rotating flow of an upper convicted Maxwell fluid over a starching sheet. commun. Theor Phys 56: $740-744$.

30. http://numericaltank.sjtu.edu.cn/BVPh20.htm

31. Sahoo B , Labropulu F (2012) Steady Homann flow and heat transfer of an electrically conducting second grade fluid. Computers and Mathematics with Applications 63: 1244-1255.

32. Imayama S (2012) Technical Reports from Royal Institute of Technology, Linn'e FLOW Centre, KTH Mechanics SE-100 44 Stockholm, Sweden. 